\title{
Programa de normalización de estudios previos y control de calidad de las intervenciones. Propiedades mecánicas. $1^{\text {a }}$ parte
}

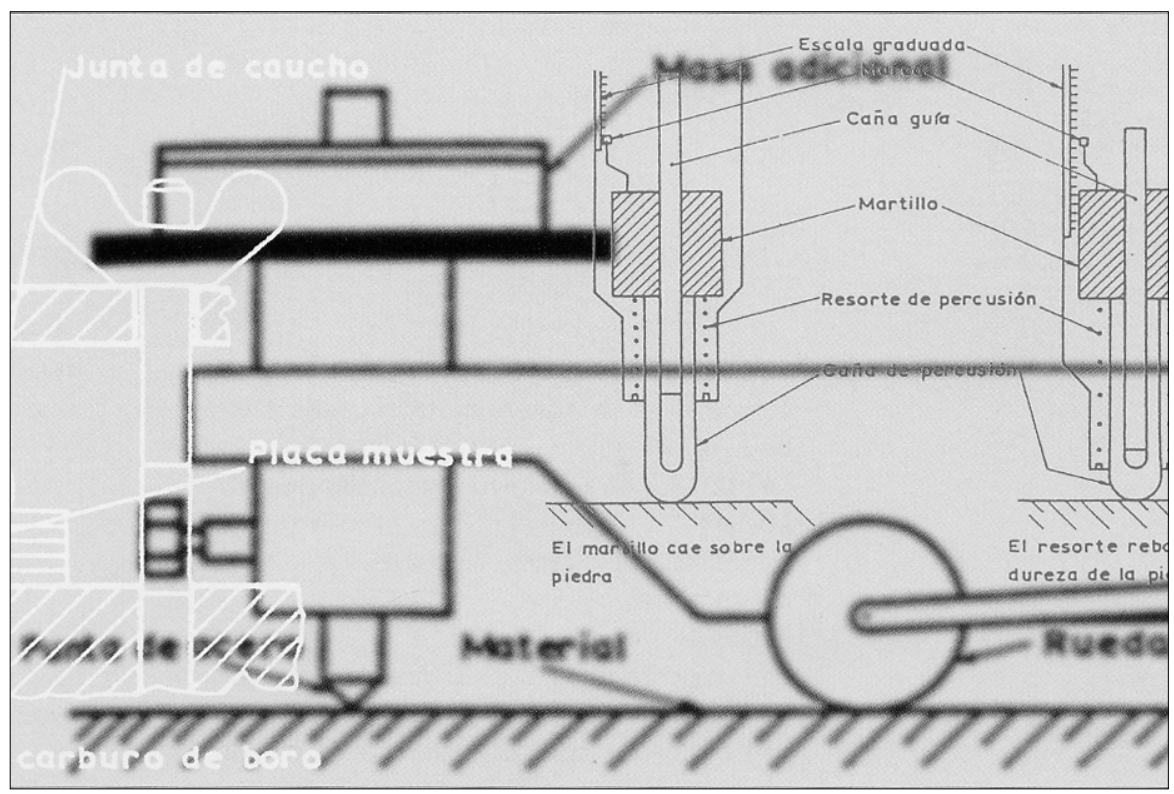

\section{Esther Ontiveros Ortega}

Rosario Villegas Sánchez

Centro de Intervención del IAPH

\section{INTRODUCCIÓN}

Dentro del Subprograma de Normalización de Estudios Previos y Control de Calidad en las Intervenciones y siguiendo en el apartado de caracterización del material, a continuación se aborda el tema de propiedades mecánicas (figura I).

El estudio del comportamiento de los materiales requiere un conocimiento a fondo de la mecánica de los sólidos, en el que se incluye además ideas básicas sobre el equilibrio y la deformación de los materiales.
Al estudiar la mecánica de los sólidos hay que tener en cuenta dos conceptos básicos: resistencia y rigidez. El método más común para evaluar estas propiedades es controlar lo que el material se deforma bajo la acción de una carga determinada.

Antes de entrar en la descripción de los ensayos para la determinación de las propiedades mecánicas de los materiales es conveniente definir dos conceptos: carga y deformación.

Cargar un material es aplicar una fuerza sobre una determinada superficie y en cierta dirección. Cuando sobre un cuerpo se aplica una carga perpendicular a la superficie, ya sea de tracción o compresión en ambos casos la carga se denomina tensión y se designa con el símbolo $\sigma$. Si las fuerzas se dirigen paralelamente a las caras del objeto, se produce una carga de cizalladura que se designa como $\tau$. En todos los casos sus dimensiones son: Fuerza/superficie. 


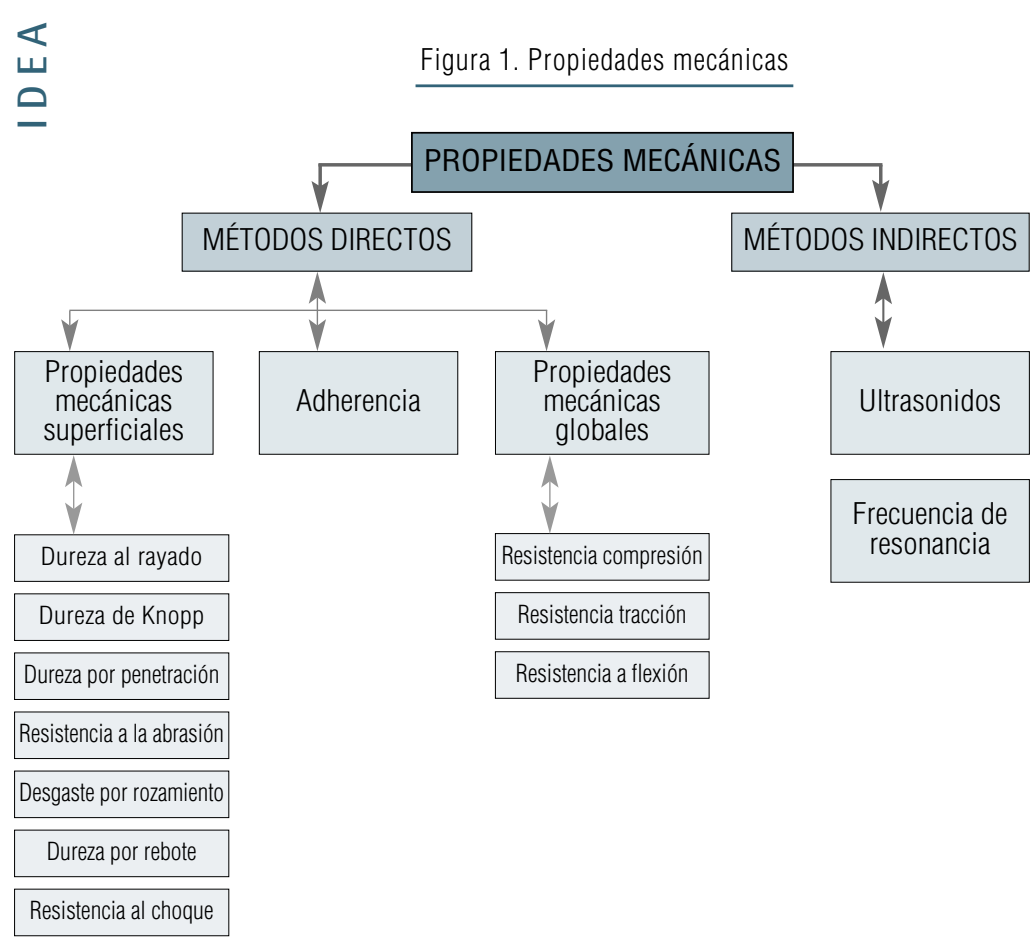

Al aplicar una fuerza sobre un cuerpo éste puede comportarse de manera dúctil (si queda deformado después del esfuerzo) o frágil (si se deteriora visiblemente). Por otra parte los materiales pueden comportarse de manera elástica, si el sólido vuelve a su estado inicial o plástica, si no vuelve a su estado inicial pero sin romperse.

En el régimen elástico existe una dependencia lineal entre la fuerza ejercida y la deformación que se denomina ley de Hooke. El coeficiente de proporcionalidad recibe diversos nombres según sea la carga y la deformación producida:

$$
\sigma_{\mathrm{x}}=\mathrm{E} \cdot \mathrm{e}_{\mathrm{x}}
$$

Donde $\sigma_{\mathrm{x}}$ es la tensión y $\mathrm{E}$ el módulo de elasticidad.

a) Módulo de Young (Y) o módulo de elasticidad (E). Se aplica para tensiones o deformaciones paralelas a las caras.

b) Cociente de Poisson para tensiones o deformaciones perpendiculares.

$$
\sigma_{x}=-\frac{E}{v} e_{y}
$$

Donde $\sigma_{\mathrm{x}}$ es la tensión aplicada, E es el módulo de elasticidad y $\mathrm{v}$ el coeficiente de Poisson.

c) Deformaciones $\lambda$ debidas a fuerzas de cizalladura se relacionan mediante el módulo de rigidez, G.

$$
\tau=\mathrm{G} \lambda
$$

Donde $\tau$ es carga de cizalladura, $\mathrm{G}$ módulo de rigidez y $\lambda$ deformación.
El valor mínimo de carga capaz de producir una deformación plástica se denomina limite elástico $\sigma_{\mathrm{c}}$.

El comportamiento físico-mecánico de un material está directamente relacionado con su microestructura (textura y/o estructura) y ésta a la vez va a depender de los siguientes factores:

- Características petrográficas; que hace referencia a la resistencia de cada uno de sus componentes petrográficos (minerales, clastos o granos, fósiles y el cemento que une estos elementos).

- Sistema poroso y/o fisuras que presentan.

- La presencia de agua en el seno poroso del material, debido a que el agua provoca una reducción de la energía en la superficie de contacto entre los diferentes elementos en el material, modifica las uniones intergranulares, por la presión de las mismas en el seno poroso; provoca fenómenos de stress-corrosión y favorece la propagación de fisuras.

Además a la hora de estudiar el comportamiento mecánico de un material usado en construcción hay que abordar otros dos aspectos:

- Su comportamiento estático en relación a la forma, modalidad de puesta en obra y funcionalidad de cada elemento en la estructura.

- Comportamiento dinámico en relación a las condiciones microclimaticas del entorno (por ejemplo oscilaciones térmicas, etc.).

Desde el punto de vista mecánico, a la hora de definir un material, hay que tener en cuenta dos aspectos: el comportamiento superficial y el comportamiento como un todo frente a las fuerzas mecánicas externas.

Las propiedades mecánicas superficiales de los materiales pétreos están ligadas a la dureza superficial o resistencia superficial a la deformación.

Las propiedades mecánicas globales se valoran a través de ensayos directos que miden la fuerza crítica que provoca la ruptura de una probeta (resistencia a comprensión, flexión y tracción) o bien mediante determinados ensayos indirectos que permiten calcular el módulo dinámico de elasticidad, en función de la velocidad de propagación del sonido o de la frecuencia de resonancia.

Como ensayos intermedios entre los superficiales y globales se encuentran los ensayos de adherencia, que miden la adhesión o falta de cohesión entre una capa externa más o menos profunda y el substrato.

En este artículo se abordan los ensayos para la determinación de las propiedades mecánicas superficiales y de adherencia. 


\section{COMPORTAMIENTO MECÁNICO SUPERFICIAL}

Las propiedades mecánicas superficiales de los materiales están ligadas a la dureza superficial y podría considerarse como el comportamiento frágil o plástico de un material pétreo frente al impacto o fragilidad. Los ensayos que se utilizan para definir esta propiedad son los siguientes:

\section{I Dureza o resistencia al rayado (dureza mineralógica)}

Se basa en la propiedad que tienen todos los materiales a rayar a otro o a ser rayado por él. Los ensayos que se han definido para determinar esta propiedad están en función de la presión requerida para realizar un rayado o el tamaño de rayado producido por un estilete o punta que corre sobre la superficie del material bajo una carga fija.

Este ensayo se realiza habitualmente en laboratorio, la medida no es muy precisa, requiriendose un gran número de medidas en una misma probeta. La limitación de esta técnica está en que no se puede aplicar sobre materiales demasiado duros, por ejemplo, granitos.

Los ensayos de dureza por rayado son difíciles de normalizar y para su determinación se ha empleado el aparato de Martens.

\section{Procedimiento de ensayo (de Martens)}

Características del aparato. El aparato se compone de un pequeño carro que descansa sobre dos ruedas y una punta (de acero tratada o punta de diamante de forma piramidal), cuyo ángulo en el vértice es de $90^{\circ}$, y sobre la cual, mediante un brazo de palanca, se hace actuar una carga determinada. La probeta queda sujeta en un soporte adecuado y sobre ella se desplaza la punta de raya (figura 2).

Preparación de las muestras. Se requiere probetas en forma cúbica de 4 a $8 \mathrm{~cm}$ de diámetro. El ensayo debe realizarse en un mínimo de 3 a 5 probetas por litotipo y éstas se deben situar controlando los planos de estratificación o/y anisotropías del material.

Descripción del ensayo. Las muestras se cubren con negro de humo y posteriormente se sitúan sobre su soporte, de manera que el rayado sea perpendicular a la estrataficación o anisotropía del material. Posteriormente se procede al rayado de la muestra moviendo con la ayuda del mango el carro cargado con un peso de $3 \mathrm{Kg}$. De esta forma se marca una raya cuya visualización se ve favorecida por el contraste entre el negro de humo y el color claro de la piedra. Con un micrómetro y con la ayuda de una lupa se mide la anchura de la raya.

Resultados. Se calcula la media de un total de cien medidas por litotipo llevadas a cabo sobre un número mínimo de 5 probetas.

\subsection{Dureza Knoop}

La dureza de Knoop se determina mediante un penetrador de diamante (produce huellas rómbicas) que incide en varios puntos de las muestra estableciendo la relación existente entre la carga que actúa sobre el penetrador y la huella obtenida.

\section{Procedimiento de ensayo}

Características del aparato. El aparato empleado es un microdurómetro constituido por una mesa portamuestras, un penetrador intercambiable compuesto de un mecanismo para la aplicación gradual de la carga y un microscopio con micrómetro para la medida de la huella.

Preparación de las muestras. El ensayo se efectúa sobre un mínimo de 4 muestras de $12 \times 5 \times 1 \mathrm{~cm}$. La superficie de una de las caras de la muestra se pule con la ayuda de un abrasivo de carborundum y alúmina.

\section{Figura 2. Esclerómetro de Martens}

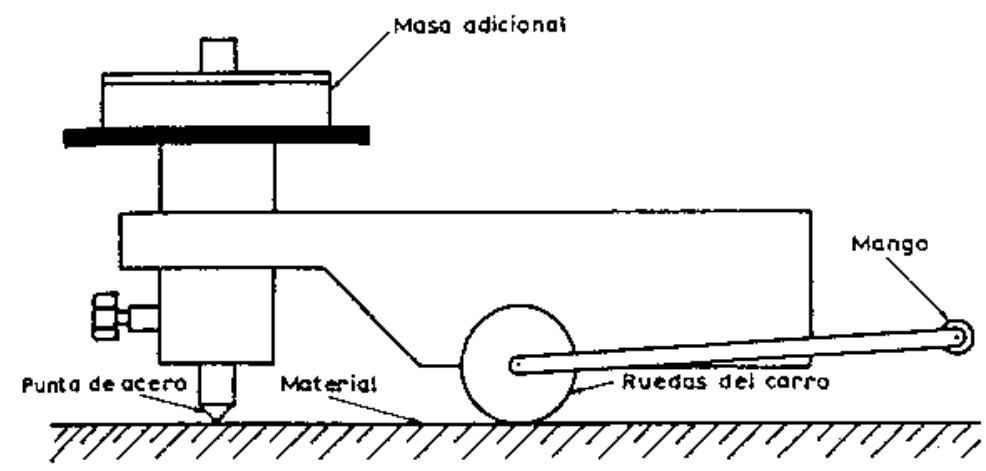

Descripción del ensayo. La muestra ya pulida se sitúa en el portamuestras del microscopio, con cera de modelar. Mediante una prensa manual se consigue el paralelismo entre la cara que recibirá la huella y el portamuestras. Seguidamente se sitúa la muestra bajo el plano del durómetro y se efectúan 20 determinaciones, utilizando una carga de $100 \mathrm{~g}$ a lo largo de dos alineaciones, distantes $2 \mathrm{~cm}$ y paralelas a la arista mayor de la probeta. Cada una de las determinaciones sobre una misma alineación estará distanciada $\mathrm{I} \mathrm{cm}$ una de otra. Finalmente se mide la longitud de la diagonal mayor de cada una de las huellas resultantes.

Resultados. La microdureza de Knoop se expresa en mega-pascales, y se calcula mediante la siguiente fórmula matemática:

$$
\mathrm{DK}=139.454 \frac{\mathrm{P}}{\mathrm{L}^{2}}
$$

Donde $\mathrm{P}$ es la carga del penetrador (g) y

$\mathrm{L}$, la longitud de la diagonal mayor de cada huella $(\mathrm{mm})$ 
$\longleftarrow$
$\square$
Para obtener la microdureza de Knoop de cada muestra se hallará la media aritmética de las microdurezas obtenidas aplicando la formula anterior de las 20 mediciones efectuadas. Se tomará como resultado del ensayo la media aritmética de las microdurezas Knoop del conjunto de 4 muestras.

\subsection{Ensayos de penetración}

Estos ensayos de microdureza consiten en marcar la superficie del material con el apoyo de una punta. Según la forma geométrica de la punta y el método de aplicación de la carga, se distinguen varios tipos de ensayos:

Método de Brinell. En este caso se utiliza una bola esférica sobre la que se aplica una carga constante.

Método de Vickers. Se utiliza una punta piramidal de diamante (con ángulo entre las caras de $136^{\circ}$ ) y el valor de la carga es constante durante el tiempo del ensayo.

Método de Rockwell. Es similar a los anteriores, la diferencia radica en que la punta de diamante tiene forma cónica (con un ángulo de $120^{\circ}$ ) y la punta está redondeada circularmente (radio $0.2 \mathrm{~mm}$ ).

Resultados. En todos los métodos el valor de la microdureza se determina como el cociente entre la carga aplicada y la proyección sobre la superficie de la muestra de la huella dejada por la punta. Debido a las peculiaridades del proceso experimental se considera que el valor del límite elástico medido a través de pruebas convencionales de comprensión o tracción es un tercio del valor de la microdureza, el resto de la presión ejercida durante el ensayo de microdureza solo contribuye a la deformación de la red.

Figura 3. Esquema del aparato empleado para la determinación de la dureza por abrasión

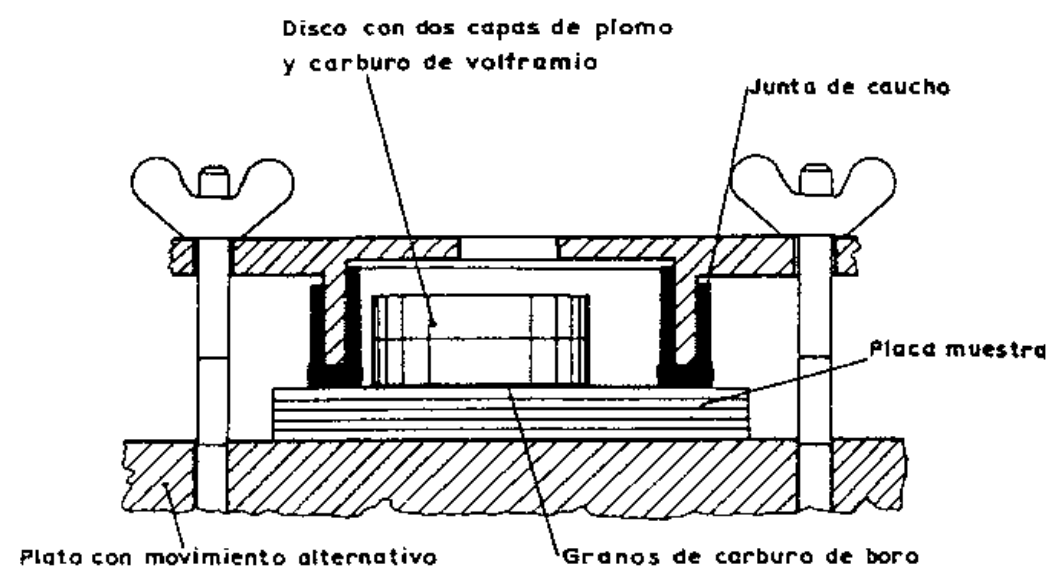

\subsection{Dureza por desgaste o resistencia a la abrasión}

Esta magnitud se utiliza para conocer la resistencia de un material a las pisadas, y va a estar en función de la dureza media de los componentes mineralógicos del material y la fortaleza del enlace interfacial.

Para normalizar el ensayo de dureza por abrasión se ha seguido el método propuesto por el Instituto de Materiales de Berlín que es una modificación del aparato diseñado por PEI (norma ASTMC/448-64).

\section{Procedimiento de ensayo}

Descripción del ensayo. En la figura 3 se representan de forma esquemática las partes fundamentales del aparato. La muestra, situada sobre un plato con movimiento alternativo, es sometida a la acción abrasiva de un disco que contiene dos capas de plomo y carburo de volframio. Los granos de abrasivo deben ser por término medio no sustancialmente más pequeños que los granos estructurales, las partículas abrasivas o los poros del material.

Preparación de las muestras. Las probetas deben cortarse en forma de placa cuya superficie debe ser plana y no demasiado friable. A la hora de situar las probetas, como en el caso anterior, hay que tener cuidado con los planos de estratificación del material o/ y las anisotropias.

Una vez situada la muestra bien sujeta a una tabla oscilante del aparato, se procede a someter la misma a un movimiento excéntrico y horizontal. El disco plano de carburo de volframio da lugar a un progresivo desgaste de la superficie de la muestra. El número elegido de revoluciones del eje excéntrico del aparato para un ciclo de desgaste, debe adaptarse a las probetas ensayadas. Para ello es necesario que la proporcionalidad lineal del método no disminuya como consecuencia de una reducción de los granos de carburo de boro o una excesiva dilución del abrasivo por la sustancia desgastada.

Resultados. Con este ensayo se determina el cociente entre la profundidad de abrasión de la probeta y el número de revoluciones del eje excéntrico.

\subsection{Resistencia al desgaste por rozamiento}

Se denomina resistencia al desgaste por rozamiento, la resistencia que opone la superficie del material al ser sometido a un desgaste lineal producido sobre tres caras de dos probetas. Para su normalización se ha seguido la normas UNE 22-173/85.

\section{Procedimiento de ensayo}

Características del aparato. El aparato consta de una pista de rozamiento con un radio mínimo interior de 25 $\mathrm{cm}$ y de radio mínimo exterior de $40 \mathrm{~cm}$ capaz de girar a una velocidad mínima relativa de $1 \mathrm{~m} / \mathrm{s}$, referido al centro de la probeta. Consta de dos portaprobetas, solidarios a los ejes de deslizamiento y diametralmente 
opuestos sobre el bastidor, que están centrados sobre la circunferencia media de las pistas de rozamiento. Presenta un dispositivo mediante el cual se puede comprimir una probeta entre los platos con una presión de $0.0588 \mathrm{MPa}$, otro dispositivo que permita verter el abrasivo y agua en la superficie de rozamiento.

Preparación de las muestras. Se preparan probetas de 7 $\mathrm{cm}$ de arista, con una tolerancia de 5\%. Se determina su volumen inicial por pesada hidrostática $\left(\mathrm{PH}^{\circ}{ }^{\circ} 22\right)$, y la superficie a desgastar midiendo con un calibre (precisión $0.1 \mathrm{~mm}$ ) las dimensiones de las caras. La medida tomada es la media entre las aristas y el centro de las caras. El ensayo se hace sobre un mínimo de dos probetas.

Descripción del ensayo. Una vez colocadas las muestras en los portas se carga a razón de $0.0588 \mathrm{MP}$, la máquina se pone en marcha y se va vertiendo, de manera uniforme, el abrasivo (carborundum) cuyos tamaños de granos deben estar comprendidos entre los tamices 0.33 (UNE 7-050) y 0.63 (UNE 7-050) en una cantidad de $1 \mathrm{~g} / \mathrm{cm} 2$ de la superficie de la mayor cara de las sometidas al desgaste, así como 12 gotas de agua por minuto. Se someten las probetas a un recorrido de 1000 m, sacándolas posteriormente de la máquina y limpiandolas cuidadosamente.

Resultados. El desgaste lineal ( $\mathrm{mm}$ ) se determina con la siguiente expresión:

$$
\mathrm{D}=\frac{\mathrm{V}_{\mathrm{i}}-\mathrm{V}_{\mathrm{f}}}{\mathrm{A}}
$$

Donde: $V_{i}$ es el volumen inicial

$V_{f}$ es el volumen final

A superficie de las caras de las probetas en contacto $(\mathrm{mm})$.

Se toma como resultado definitivo la media aritmética de los desgastes lineales de cada una de las dos probetas con un error inferior de $0.1 \mathrm{~mm}$.

\subsection{Dureza por rebote}

Dentro de los ensayos dinámicos, los de mayor interés son los de rebote o retroceso, basados en el hecho de que si se deja caer desde una altura un pequeño martillo de punta redondeada, la energía de caída es absorbida por el material en forma de una huella parcialmente elástica. El martillo rebota elevandose a una altura inferior a la de su partida, y esta altura se toma como medida de la dureza por rebote.

Existen diversos aparatos propuestos cuyos diferencias estriban en el peso del martillo, la forma de la punta y la altura de la caída. Los más conocidos son el esclerómetro de Shore y el durómetro. Los instrumentos están diseñados para actuar en posición horizontal y trabajar sobre superficies horizontales o poco inclinadas. La corrección de los datos se lleva a cabo con la ayuda de unas tablas que acompañan al aparato en concreto.

\section{Procedimiento de ensayo}

Características del aparato: (método del esclerómetro). En la figura 3 aparece un esquema del principio de un aparato utilizado para piedras blandas y en la figura 4 el esquema de uno empleado para piedras duras, su fundamento es el mismo pero su manera de actuar es diferente. En el primer caso el martillo gira sobre un eje y en el segundo caso el martillo es guiado dentro de un tubo.

Figura 4. Esclerómetro para piedras blandas
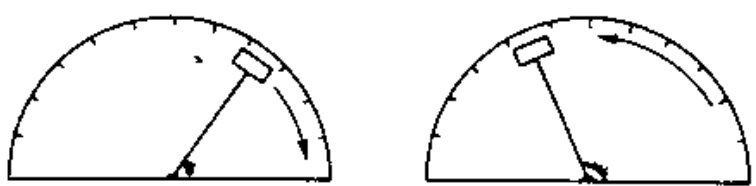

Figura 5. Esclerómetro para piedras duras

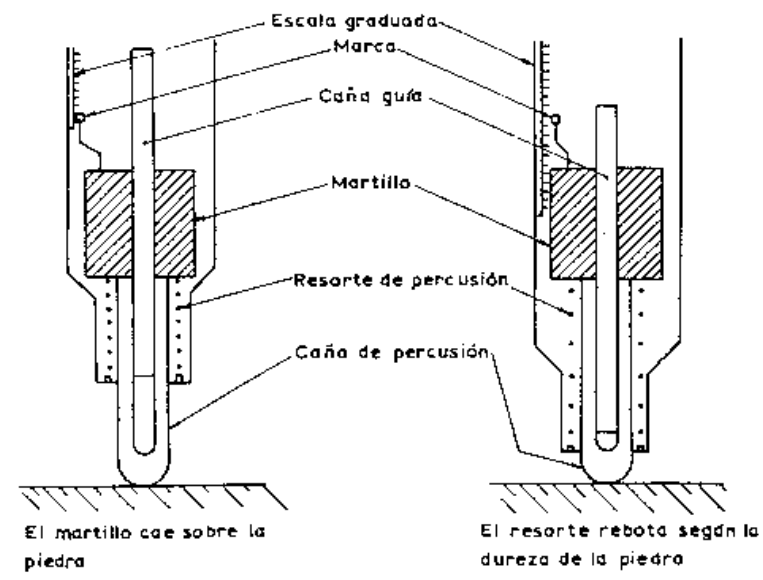

Preparación de las muestras. Este ensayo se realiza sobre material in situ pero hay que tener en cuenta que la superficie debe ser lo más lisa posible. En el laboratorio las medidas se realizarán sobre una superficie de $20 \times 20 \mathrm{~cm}$ y sobre 5 probetas diferentes dentro de un mismo litotipo.

Resultados. Los cálculos se realizan determinando la media de todas las medidas, se debe indicar el tamaño de las probetas y el número de medidas efectuadas.

Además de las medidas directas, este ensayo se puede utilizar para obtener las curvas de calibrado que dan la relación entre la resistencia a la compresión y los índices esclerométricos para tipo de material.

\subsection{Resistencia al choque}

La resistencia al choque se define como la resistencia que opone un material al impacto de un objeto sobre su superficie. Esta se determina el ensayo descrito a continuación, sometiendo las muestras al efecto de la caída vertical de una masa esférica de un peso determinado a distintas alturas. 


\section{Procedimiento de ensayo}

Características del aparato. El dispositivo esta compuesto por los siguientes elementos:

- El elemento de guiado de la masa que va a efectuar el impacto y de control de alturas desde la que se deja caer.

- El lecho de arena de $10 \mathrm{~cm}$ de espesor colocado en una caja de madera cuadrada cuyo perímetro interno debe estar distante un mínimo de $10 \mathrm{~cm}$ de la muestra.

- Y por último dos esferas de acero de 1.000 y $250 \mathrm{~g}$ respectivamente.

\section{Figura 6. Esquema del aparato de medida de adherencia}

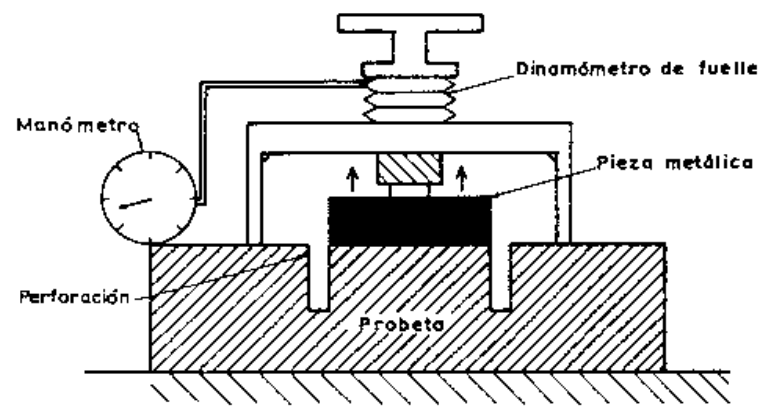

Se colocan cada una de las muestras sobre el lecho de arena, a continuación se deja caer la esfera de acero en el centro de la muestra, desde una altura inicial de $5 \mathrm{~cm}$. Posteriomente se incrementa gradualmente la altura de caída en $5 \mathrm{~cm}$ cada vez, finalizando el ensayo cuando se produce la rotura de la muestra, y en cualquier caso cuando la altura de caída ha alcanzado los $150 \mathrm{~cm}$.

Preparación de las muestras. El ensayo se hace sobre un mínimo de 4 probetas de material de dimensiones $20 \times 5 \times 1 \mathrm{~cm}$.

Resultados. La resistencia al choque sera la altura, en centímetros, en la que se produce la rotura de la muestra. Si ha resistido el ensayo sin romperse se tomará co- mo resultado la altura máxima de caída de $150 \mathrm{~cm}$. El resultado final será la media aritmética de las resistencias al choque del conjunto de muestras.

\section{ADHERENCIA}

Este ensayo mide la adhesión entre dos materiales permitiendo estimar la resistencia a la tracción de materiales pétreos además de su durabilidad, extensión de la alteración y efectividad de un tratamiento de impregnación. Para la descripción del ensayo se ha seguido la norma Rilem nㅇll.3.

\section{Procedimiento de ensayo}

Características del aparato. El equipo empleado consta de un dispositivo de tracción, un dinamómetro y una cortadora de diamante con un diámetro interno de $5 \mathrm{~cm}$, movida por una máquina rotatoria de perforación (figura 6).

Preparación de las muestras. La muestra se corta circularmente hasta la profundidad que se considere apropiada para alcanzar el objetivo propuesto, con la ayuda de una cortadora de diamante con un diámetro interno de $5 \mathrm{~cm}$, movida por una máquina rotatoria de perforación. Este ensayo debe realizarse al menos sobre tres probetas diferentes del mismo litotipo.

Descripción del ensayo. Una vez perforada la muestra se encola una pieza metálica de $5 \mathrm{~cm}$ de diámetro en el área delimitada por la huella de la perforación. Se une la pieza metálica al dispositivo de tensión a través de un tornillo de cabeza esférica. Se aplica la fuerza de tracción hasta que se produzca la ruptura de la probeta.

Resultados: Se anota la carga máxima que soporta la probeta durante el ensayo. La fuerza de ruptura, expresada por la relación entre la adhesión y el área de la pieza metálica, viene dada en Mpa. En los resultados se incluye los valores individuales para cada perforación y el valor medio de los resultados para cada profundidad de penetración.

\section{Bibliografía}

ALBELLA, JM; CINTAS, AM; MIRANDA, T; SERRACOTA, JM. Introducción a la ciencia de los materiales. Propiedades mecánicas de los Materiales. pp. 23I-252. Madrid: CSIC, 1993.

MARTíN A. Ensayos y experiencias de alteración en la conservación de obras de piedra de interés Histórico-Artístico. Características mecánicas, térmicas y eléctricas. pp. 339-369.

Normas UNE. Granitos ornamentales:

UNE 22-179-85. Resistencia al choque.

UNE 22-173-85. Resistencia al desgaste por rozamiento.

UNE 22-178-85. Microdureza de Knoop.
Normas Rilem, Recommandation provisoires - Tentative Recommendation:

Tests $N^{\circ}$ III. 2. Determination of the dynamic modulus of elasticity by pulse velocity. pp. 219-221.

Tests $\mathrm{N}^{\circ}$ III.3. the pull-out tets. pp. 223.

IV. Tests determining the mechanical surface propeties. Pp. 229-233. 